\title{
Prediction of Hemodynamic Conditions by Noninvasive Arterial Tonometry in Mitral Stenosis
}

\author{
Yukihiro Hojo, MD, Hitoshi EbaTa, MD, \\ Hiromichi SeKigughi, MD, Masanori Yamasawa, MD, \\ Hideyuki FujIKawa, MD, Toshio Kuroda, MD, \\ Uichi IKEDA, MD, \\ and Kazuyuki SHIMADA, MD
}

\section{SUMMARY}

To predict the hemodynamic conditions in patients with mitral stenosis (MS), continuous blood pressure responses were monitored noninvasively at the bedside by arterial tonometry during the Valsalva maneuver in $18 \mathrm{MS}$ patients aged $54.2 \pm 9.1(40 \sim 77)$ years ( 6 men, 12 women). Two indices during the Valsalva maneuver (blood pressure decline value at phase III (BPdec) and subsequent blood pressure overshoot value at phase IV (BPov)) were compared with hemodynamic data obtained by the cardiac catheterization method, and the correlations between the changes in these parameters were examined.

In these 18 patients, BPdec showed a significant negative correlation with the mean diastolic pressure gradient between the left atrium and left ventricle and showed a significant negative correlation with pulmonary capillary wedge pressure (PCWP) $(r=-0.62, p<0.01, r=-0.53, p<0.05$, respectively). Mitral valve area (MVA) showed a significant positive correlation with BPdec $(r=+0.63, p<0.01)$. Similarly, BPov showed a significant positive correlation with cardiac output $(\mathrm{CO})$, cardiac index $(\mathrm{CI})$ and MVA $(r=+0.60, p<0.01$, $r=+0.64, p<0.01, r=+0.65, p<0.01$, respectively $)$.

Thus, continuous monitoring of blood pressure by arterial tonometry during the Valsalva maneuver is useful for predicting the hemodynamic conditions in patients with MS. (Jpn Heart J 1966; 37: 343-352)

\section{Key words: Mitral stenosis Arterial tonometry Valsalva maneuver}

\footnotetext{
CINCE 1944 the Valsalva maneuver has been applied to the evaluation of hemodynamic conditions such as valvular heart disease ${ }^{1)}$ and the severity of congestive heart failure. ${ }^{2-5)}$ Goldberg et al reported a lack of "blood pressure overshoot" after the strain phase of the Valsalva maneuver in patients with mitral stenosis (MS) during continuous blood pressure monitoring. ${ }^{11}$ Thus the severity of

From the Department of Cardiology, Jichi Medical School, Tochigi, Japan.

Address for correspondence: Yukihiro Hojo, MD, Department of Cardiology, Jichi Medical School, Yakushiji, Minamikawachi-machi, Tochigi 329-04, Japan.

Received for publication October 30, 1995.

Accepted February 19, 1996.
} 
MS can be assessed from the continuous blood pressure response during the Valsalva maneuver.

In previous studies, however, continuous blood pressure was measured directly by intraarterial catheters placed in the brachial arteries. To avoid any adverse effects of this invasive approach, an improved technique, "arterial tonometry" was recently developed for continuous noninvasive monitoring of arterial blood pressure, and its validity and reliability were confirmed by Sato et al. ${ }^{6)}$

In the present study, we applied arterial tonometry for continuous monitoring of blood pressure during the Valsalva maneuver in MS patients at the bedside and found that this noninvasive method is readily repeatable and useful for predicting the hemodynamic conditions in MS.

\section{Methods}

Subjects: To examine the validity of Valsalva indices for the prediction of hemodynamic condition in MS, 18 patients with various degrees of MS were studied. Patients were excluded if the MS was combined with other valvular diseases such as severe mitral regurgitation (MR), aortic valvular diseases or severe left ventricular dysfunction. The diagnosis and severity of MS were determined by history, physical examination, chest X-ray, two-dimensional and Doppler echocardiography and cardiac catheterization. Informed consent was obtained from each patient.

Table I shows the basic characteristics of the total study population. The patients ranged in age from 40 to 77 years with a mean \pm SD of $54.2 \pm 9.1$ years. Six of the patients were men and 12 women. Three patients were in New York Heart Association functional class I, 11 were in class II and 4 were in class III. Fourteen of the patients showed atrial fibrillation (Af) and 4 had normal sinus rhythm. MR was graded according to the criteria of Sellers et al.7): grade I $(n=8)$, grade II $(n=3)$. Subjects who had MR exceeding Sellers grade III were excluded. Mean heart rate was $75.7 \pm 17.4 \mathrm{bpm}$. There was no significant difference in heart rate between the patients with Af and those with normal sinus rhythm (71.8 \pm 6.0 vs $76.9 \pm 19.6 \mathrm{bpm})$. The mean left ventricular ejection fraction was $55.3 \pm 9.9 \%(43 \sim 74 \%)$. Mean mitral valve area (MVA) calculated by the Gorlin formula ${ }^{8)}$ and by the pressurc half-time (PHT) method $^{9)}$ was $1.15 \pm 0.71 \mathrm{~cm}^{2}\left(0.53 \sim 2.7 \mathrm{~cm}^{2}\right)$ and $0.95 \pm 0.61 \mathrm{~cm}^{2}\left(0.30 \sim 1.7 \mathrm{~cm}^{2}\right)$, respectively. Although the mean MVA values determined by the PHT method were lower than those by the Gorlin formula, there were no significant differences between them. Digitalis $(0.125 \sim 0.25 \mathrm{mg})$ was administered to 16 patients, furosemide $(20 \sim 40 \mathrm{mg})$ to 16 and warfarin potassium $(1 \sim 5 \mathrm{mg})$ to 14 . 
Table I. Basic Characteristics of the Total Population

\begin{tabular}{|c|c|}
\hline Age (years old) & $54.2 \pm 9.1(40 \sim 77)$ \\
\hline Sex (men: women) & $6: 12$ \\
\hline $\mathrm{Af}^{*}: \mathrm{NSR}^{\# \#}$ & $14: 4$ \\
\hline \multicolumn{2}{|l|}{ NYHA class } \\
\hline I & 3 \\
\hline II & 11 \\
\hline III & 4 \\
\hline \multicolumn{2}{|c|}{ Sellers' grade of mitral regurgitation ${ }^{7}$ (left ventriculography) } \\
\hline None & 7 \\
\hline I & 8 \\
\hline II & 3 \\
\hline \multicolumn{2}{|c|}{ Grade of mitral regurgitation (Doppler echocardiography) } \\
\hline None & 7 \\
\hline Mild & 9 \\
\hline Moderate & 2 \\
\hline Heart rate $(\mathrm{bpm})$ & $75.7 \pm 17.4$ \\
\hline Ejection fraction $(\%)^{*}$ & $55.3 \pm 9.9(43 \sim 74)$ \\
\hline MVA $\left(\mathrm{cm}^{2}\right)$ by the Gorlin formula** & $1.15 \pm 0.71(0.53 \sim 2.7)$ \\
\hline MVA $\left(\mathrm{cm}^{2}\right)$ by echocardiography & $0.95 \pm 0.61(0.30 \sim 1.7)$ \\
\hline
\end{tabular}

Echocardiography: Two-dimensional and Doppler echocardiography examinations (Toshiba SSH-160A, Tokyo, Japan) were performed 2 days before cardiac catheterization. Images were acquired using a $3.5-\mathrm{MHz}$ transducer, and continuous Doppler echo was performed using a 2.5- $\mathrm{MHz}$ transducer. MVA in each patient was calculated by the PHT method. The severity of MR was graded by two-dimensional color Doppler echocardiography. The regurgitant flow was evaluated from a parasternal long-axis and apical two-and four-chamber views. The grade of MR was divided into three groups (mild, moderate, severe). ${ }^{10}$ )

Gardiac catheterization: All MS patients underwent diagnostic left and right heart catheterization. Left ventriculography was performed using a $6 \mathrm{Fr}$ pigtail catheter via the right femoral artery, and the left ventricular ejection fraction and degree of MR were evaluated. Right heart catheterization was performed using a 7 Fr balloon flotation (Swan-Ganz) catheter via the right femoral vein. The pressures at the inferior vena cava, right atrium, right ventricle, pulmonary artery, pulmonary capillary, left ventricle (LV) and aorta were obtained using a fluid-filled catheter system. Simultaneous pressures were measured to obtain the mean diastolic pressure gradient between the left atrium (LA) and LV. Cardiac output $(\mathrm{CO})$ and cardiac index (CI) were measured by the thermodilution method and MVA was calculated according to the Gorlin formula.

Valsalva maneuver and continuous blood pressure measurements: At the bedside, each subject performed the Valsalva maneuver by expiring forcefully 


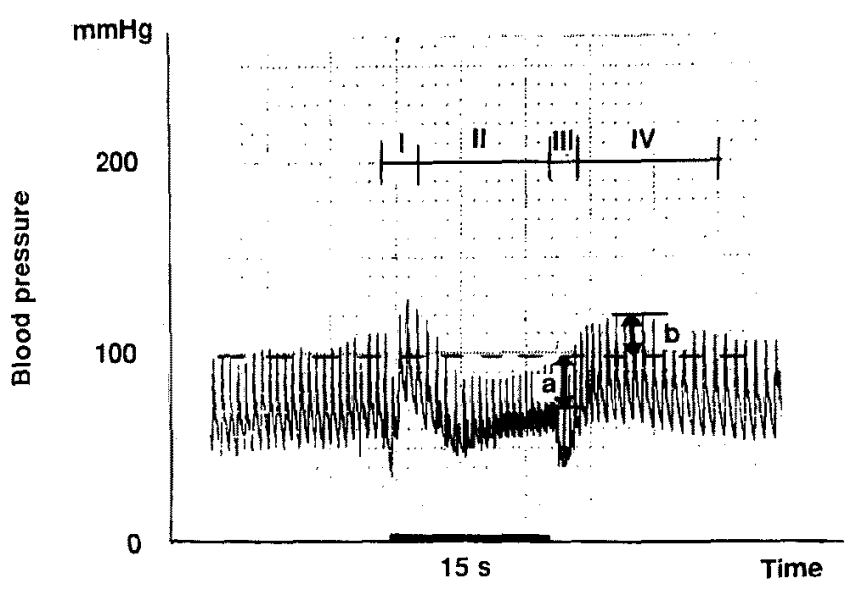

Figure 1. A normal blood pressure response during the Valsalva maneuver recorded by arterial tonometry. The figure shows a representative tonometry record of blood pressure response during the Valsalva maneuver. Vertical axis shows the blood pressure. Paper speed was $50 \mathrm{~mm} / \mathrm{min}$. a: blood pressure decline at Valsalva phase III, b: blood pressure overshoot at Valsalva phase IV. Each index was standardized according to resting systolic blood pressure.

into a rubber tube connected to a mercury column and maintaining a constant airway pressure of $40 \mathrm{mmHg}$ for $15 \mathrm{sec}$ in a supine position according to the method described by Knowles et al. ${ }^{11}$ Before measuring the continuous blood pressure, casual blood pressures were measured at both arms in a supine position after a 10-min rest. Patients with a blood pressure difference of more than 10 $\mathrm{mmHg}$ between the right and left arms were excluded from the study. The continuous systemic arterial pressure was measured by percutaneous arterial tonometry (CBM 7000, Nippon Kolin, Tokyo) at the left or right radial artery. Details of the method were described by Sato et al. ${ }^{6}{ }^{6}$ Briefly, the arterial tonometry sensor was placed on the radial side of the extended wrist and the pressure pulse was detected. The recording was carried out at a paper speed of $50 \mathrm{~mm} / \mathrm{min}$. The blood pressure was measured continuously after an additional 10-min rest. Each subject practiced the Valsalva maneuver at least 3 times until a clear pressure response curve was obtained. After practicing, the blood pressure responses during the Valsalva maneuver were recorded at least 3 times in patients with normal sinus rhythm, and the mean value of each index was employed. In patients with Af, responses were recorded at least 10 times because they showed a systolic blood pressure variation according to the previous R-R interval. The electrocardiogram was recorded simultaneously at the limb leads. A typical arterial pressure response during the Valsalva maneuver in a normal individual is shown in Figure 1, where the blood pressure response is divided into four phases, as suggested by Hamilton et al. ${ }^{12)}$ The two indices of circulatory 
response were defined as blood pressure decline at phase III (BPdec) and subsequent blood pressure overshoot at phase IV (BPov). Because both of the Valsalva indices were affected by systolic blood pressure, ${ }^{13)}$ they were normalized according to resting systolic blood pressure. There was no significant difference in Valsalva indices between subjects with lone Af and subjects with normal sinus rhythm. Thus the effect of Af on Valsalva indices was considered to be negligible.

Among the 18 MS patients, 9 had a mild degree of MR and 2 had a moderate degree. Therefore, in order to examine the effect of MR on Valsalva indices, 10 normal controls and 15 patients with mild to severe MR underwent the same examination. Patients with other valvular diseases or severe left ventricular dysfunction were excluded.

Data analysis: Data are expressed as mean \pm SD. Differences at $p<0.05$ were considered to be significant. The linear regression line was calculated by the least squares method to evaluate the correlation between the Valsalva index and hemodynamic parameters. The significance of the relationship between the variables was calculated using the two-tailed test of probability. Differences in the values of the Valsalva index among the MR groups were compared by one-way analysis of variance (ANOVA) followed by the Bonferroni test.

\section{Results}

Valsalva indices used to predict hemodynamic data in MS patients: Figure 2 shows representative blood pressure responses determined by arterial tonometry during the Valsalva maneuver in patients with mild or severe MS. In the patients with severe MS, the values of BPdec and BPov were decreased, as reported by Goldberg et al.") Table II shows the correlation between Valsalva indices and hemodynamic parameters obtained by cardiac catheterization. The mean diastolic pressure gradient between $\mathrm{LA}$ and $\mathrm{LV}\left(\mathrm{PG}_{\mathrm{LA} / \mathrm{LV}}\right)$ and pulmonary capillary wedge pressure (PCWP) showed a significant negative correlation with BPdec $(r=-0.62, p<0.01, r=-0.53, p<0.05$, respectively). The systolic, diastolic and mean pulmonary arterial pressure also showed a significant negative correlation with BPdec $(r=-0.64, p<0.01, r=-0.50, p<0.05, r=-0.58$, $p<0.05$, respectively). The values of CO, CI and MVA determined using the Gorlin formula showed a significant positive correlation with BPdec $(r=+0.66$, $p<0.01, r=+0.63, p<0.01, r=+0.63, p<0.01$, respectively).

Furthermore, the values of $\mathrm{CO}, \mathrm{CI}$ and MVA by the Gorlin formula showed a significant positive correlation with BPov $(r=+0.60, p<0.01$, $r=+0.64, p<0.01, r=+0.65, p<0.0 \mathrm{l}$, respectively). Systemic vascular resistancc showed a significant negative correlation with BPov $(r=-0.66, p<0.01)$.

Effect of mitral regurgitation: Ten patients were used as controls (without 

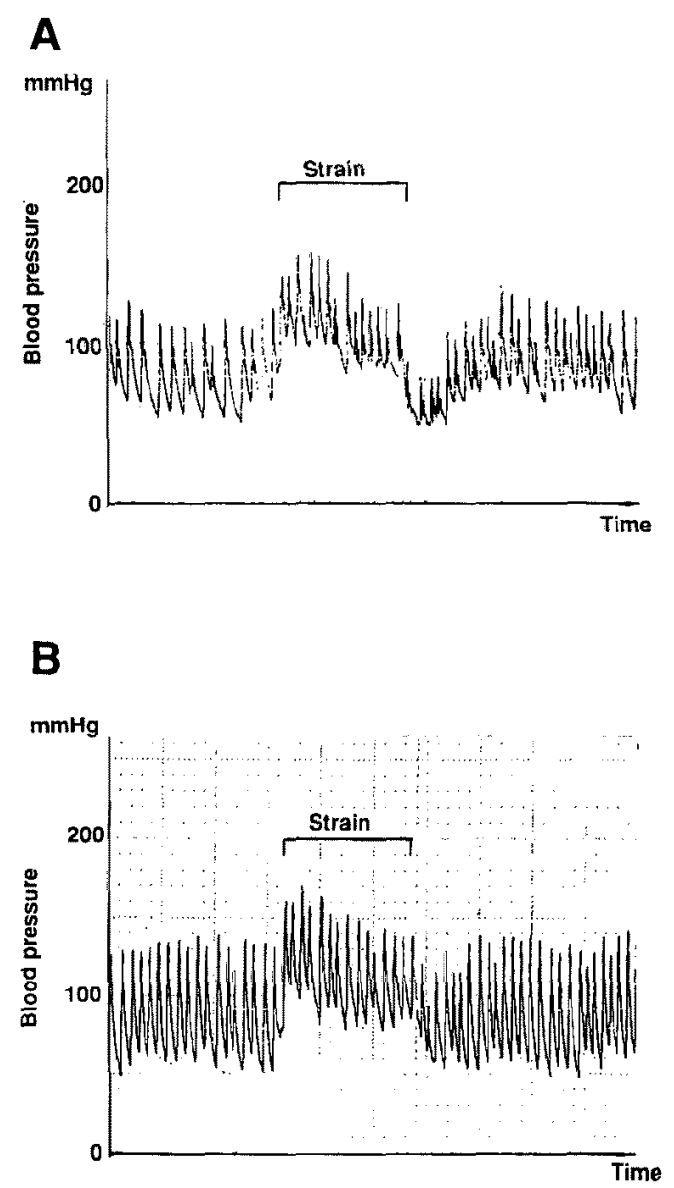

Figure 2. Representative records of blood pressure response during the Valsalva maneuver by arterial tonometry in patients with mitral stenosis. Panel A : recording of a patient with mild mitral stenosis. In this case mitral valve area was $2.3 \mathrm{~cm}^{2}$ and pulmonary capillary wedge pressure $8 \mathrm{mmHg}$. Blood pressure decline at Valsalva phase III was $32 \mathrm{mmHg}$ and blood pressure overshoot at Valsalva phase IV was 18 $\mathrm{mmHg}$. Panel B : recording of a patient with severe mitral stenosis. In this case mitral valve area was $0.9 \mathrm{~cm}^{2}$ and pulmonary capillary wedge pressure $19 \mathrm{mmHg}$. Blood pressure decline at Valsalva phase III was $10 \mathrm{mmHg}$ and blood pressure overshoot at Valsalva phase IV was $4 \mathrm{mmHg}$.

MR) and 15 patients had various degrees of MR. Their mean age was $60.0 \pm 4.1$ yr. Eleven patients were men and 14 women. As shown in Figure 3, the value of BPdec was significantly decreased only in patients with severe MR $\left(25.3 \pm 9.1 \times 10^{-2}\right.$ for control, $28.8 \pm 14.7 \times 10^{-2}$ for mild MR, $22.0 \pm 11.0 \times 10^{-2}$ for moderate MR and $7.2 \pm 4.6 \times 10^{-2}$ for severe MR, respectively). The value of BPov was also decreased only in patients with severe MR $\left(13.9 \pm 6.9 \times 10^{-2}\right.$ for control, $19.0 \pm 7.8 \times 10^{-2}$ for mild MR, $20.7 \pm 13.3 \times 10^{-2}$ for moderate MR and $5.4 \pm 5.2 \times 10^{-2}$ for severe MR, respectively). There were no significant differ- 
Table II. Correlations between Valsalva Indices and Hemodynamic Parameters

A. Correlations between blood pressure decline at phase III and hemodynamic parameters.

\begin{tabular}{lcc}
\hline \multicolumn{1}{c}{ Hemodynamic parameters } & $\begin{array}{c}\text { Correlation coefficient } \\
(r)\end{array}$ & $p$ (two tailed, \%) \\
\hline Pressure gradient between IA and LV $(\mathrm{mmHg})$ & -0.62 & $0.01^{*}$ \\
Pulmonary capillary wedge pressure $(\mathrm{mmHg})$ & -0.53 & $0.05^{*}$ \\
Systolic pulmonary arterial pressure $(\mathrm{mmHg})$ & -0.64 & $0.01^{*}$ \\
Diastalic pulmonary arterial pressure $(\mathrm{mmHg})$ & -0.50 & $0.05^{*}$ \\
Mean pulmonary arterial pressure $(\mathrm{mmHg})$ & -0.58 & $0.05^{*}$ \\
Right atrial pressure $(\mathrm{mmHg})$ & -0.19 & 0.48 \\
Cardiac output $(l / \mathrm{min})$ & +0.66 & $0.01^{*}$ \\
Cardiac index $\left(l / \mathrm{min} / \mathrm{m}^{2}\right)$ & +0.63 & $0.01^{*}$ \\
Mitral valve area $\left(\mathrm{cm}^{2}\right)($ by the Gorlin formula) & +0.63 & $0.01^{*}$ \\
Total pulmonary resistance $\left(\right.$ dyne $\left./ \mathrm{s} / \mathrm{cm}^{5} / \mathrm{m}^{2}\right)$ & -0.50 & $0.05^{*}$ \\
\hline
\end{tabular}

$n=18 . \mathrm{LA}=$ left atrium; $\mathrm{LV}=$ left ventricle; * Correlation with statistical significance.

B. Correlation between blood pressure overshoot at phase $I V$ and hemodynamic parameters.

\begin{tabular}{lcc}
\hline \multicolumn{1}{c}{ Hemodynamic parameters } & $\begin{array}{c}\text { Correlation coefficient } \\
(r)\end{array}$ & $p$ (two tailed, \%) \\
\hline Pressure gradient between LA and LV $(\mathrm{mmHg})$ & -0.33 & 0.08 \\
Pulmonary capillary wedge pressure $(\mathrm{mmHg})$ & -0.34 & 0.07 \\
Cardiac output $(l / \mathrm{min})$ & +0.60 & $0.01^{*}$ \\
Cardiac index $\left(l / \mathrm{min} / \mathrm{m}^{2}\right)$ & +0.64 & $0.01^{*}$ \\
Mitral valve area $\left(\mathrm{cm}^{2}\right)($ by the Gorlin formula) & +0.65 & $0.01^{*}$ \\
Systemic vascular resistance $\left(\mathrm{dyne} / \mathrm{s} / \mathrm{cm}^{\mathrm{s}} / \mathrm{m}^{2}\right)$ & -0.66 & $0.01^{*}$ \\
\hline
\end{tabular}

$n=18 . \mathrm{LA}=$ left atrium; $\mathrm{LV}=$ left ventricle; * Correlation with statistical significance.

ences in the values of BPdec and BPov among the controls and patients with mild and moderate MR.

\section{Discussion}

The Valsalva maneuver has long been applied for the bedside evaluation of the severity of valvular heart disease, ${ }^{11}$ congestive heart failure, ${ }^{2-5)}$ left ventricular function ${ }^{14,15)}$ and autonomic nervous function. ${ }^{16)}$ There have been several reported attempts to predict hemodynamic parameters using this maneuver. Schmidt et $\mathrm{al}^{17}$ ) reported the detection of elevated left ventricular end-diastolic pressure and Zema et $\mathrm{al}^{5)}$ also reported the prediction of left ventricular dysfunction using the abnormal blood pressure response during the Valsalva maneuver. In both of their studies, the simplified method using a sphygmomanometer was employed to monitor the abnormal blood pressure response during the Valsalva maneuver. Recently, McIntyre et al reported noninvasive prediction of PCWP by blood pressure response at the finger tip using a pulse-processing system during the Valsalva maneuver. ${ }^{(8)}$

In patients with MS, Goldberg et al reported that the blood pressure re- 
A

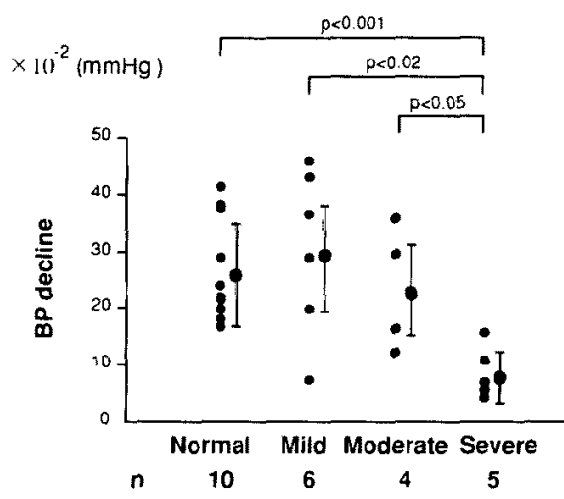

B

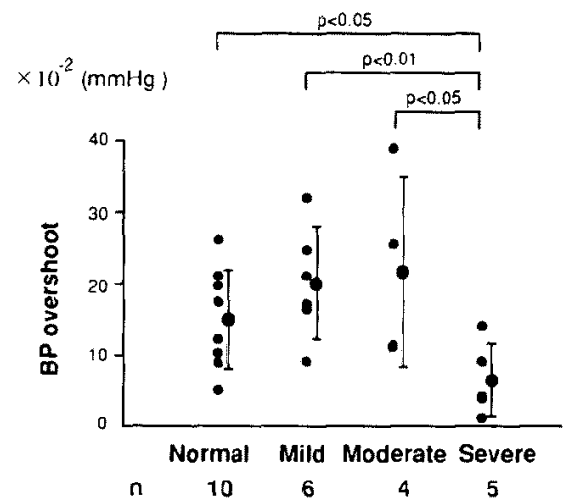

Figure 3. Effect of mitral regurgitation on Valsalva indices. Panel A shows the blood pressure decline at Valsalva phase III among the three mitral regurgitation (MR) groups and the control group. The mean values of blood pressure decline were $25.3 \pm 9.1 \times 10^{-2}$ for control, $28.8 \pm 14.7 \times 10^{-2}$ for mild MR, $22.0 \pm 11.0 \times 10^{-2}$ for moderate $\mathrm{MR}$, and $7.2 \pm 4.6 \times 10^{-2}$ for severe MR. The mean blood pressure decline in the severe MR group was significantly lower than in the other groups. Panel B shows the blood pressure overshoot at Valsalva phase IV among the three MR groups and the control group. The mean values of blood pressure overshoot were $13.9 \pm 6.9 \times 10^{-2}$ for control, $19.0 \pm 7.8 \times 10^{-2}$ for mild MR, $20.7 \pm 13.3 \times 10^{-2}$ for moderate MR, and $5.4 \pm 5.2 \times 10^{-2}$ for severe MR. The mean blood pressure overshoot in the group with severe MR was significantly lower than in the other groups. Statistical significance was examined by one-way analysis of variance.

sponse curve during the Valsalva maneuver was altered.") They reported that BPov at Valsalva phase IV was absent or minimal in patients with severe MS and speculated that this absence of BPov was due to a decrease in $\mathrm{CO}$ caused by 
impairment of early diastolic blood filling from the LA to LV through the narrowed mitral orifice. They recorded blood pressure continuously by direct insertion of a catheter into the brachial artery. Recently, noninvasive arterial tonometry has been improved, and its validation has been confirmed by Sato et al. ${ }^{6)}$ In the present study, we used this noninvasive method for monitoring blood pressure. This method was found to be simple and easy to carry out repetitively at the bedside.

We compared the value of the Valsalva indices and the data obtained by cardiac catheterization. The value of BPdec showed a significant negative correlation with $\mathrm{PG}_{\mathrm{LA} / \mathrm{LV}}$ and PGWP. In normal individuals, it was assumed that BPdec was caused by the reduction of preload due to the decrease of venous return to the heart during the strain phase and the immediate decrease of intrathoracic pressure due to the release of strain. Our results demonstrated that BPdec was minimal or absent in MS patients with pulmonary congestion. The lack of blood pressure decline was thought to be due to the minimal reduction of preload at Valsalva phase III because of excessive pulmonary vascular blood pooling in MS patients. Furthermore, the significant positive correlation between BPdec and MVA and GO was thought to be due to the decreased MVA and CO in MS patients with pulmonary congestion.

The value of the BPov showed a significant positive correlation with $\mathrm{CO}$ and MVA. Zema et al reported that the value of pressure overshoot at Valsalva phase IV showed a significant positive correlation with the left ventricular ejection fraction and a significant negative correlation with left ventricular end-diastolic pressure..$^{5)}$ The BPov was presumably defined by the $\mathrm{CO}$ and peripheral vascular resistance. In the present study, BPov was significantly positively correlated with $\mathrm{CO}$ and negatively correlated with systemic vascular resistance. Similar to the results of Goldberg et al, ${ }^{1}$ our results suggest that the decreased BPov was caused by the decrease of $\mathrm{CO}$ due to impairment of blood filling of the LV through the stenosed mitral valve.

Our results showed that in patients with severe MR, BPdec and BPov were significantly decreased in comparison with those with mild or moderate MR. Thus if severe MR is complicated, Valsalva indices would be underestimated. These results suggest that Valsalva indices are applicable for assessment of hemodynamic conditions in MS without severe MR. The MR patients with altered Valsalva indices were also confirmed to have increased PCWP and decreased CO by cardiac catheterization (data not shown).

Although there were some limitations in subject selection, our simple and noninvasive examination was considercd useful for evaluating the severity of MS at the bedside. We were able to predict not only MVA but also PCWP and CO in MS patients by applying artcrial tonometry. Further studies should be per- 


\section{formed to evaluate other valvular heart diseases or post-operative valvular heart diseases.}

\section{REFERENCES}

1. Goldberg H, Elisberg EI, Katz LN. The effects of Valsalva-like maneuver upon the circulation in normal individuals and patients with mitral stenosis. Circulation 1952; 5: 38-47.

2. Stucki $P$, Hatcher JD, Judson WE, Wilkins RW. Studies of circulation time during the Valsalva test in normal subjects and in patients with congestive heart failure. Circulation 1955; 11: 900-7.

3. Sharpey-Schafer EP. Effects of Valsalva's maneuver on the normal and failing circulation. Br Med J 1955; 19: 693-5.

4. Parisi AF, Harrington BS, Askenazi J, Pratt RC, McIntyre. Echocardiographic evaluation of the Valsalva maneuver in healthy subjects and patients with and without heart failure. Circulation 1976; 54 : $921-7$.

5. Zema MJ, Caccavano $M$, Kligfield $P$. Detection of left ventricular dysfunction in ambulatory subjects with the bedside Valsalva maneuver. Am J Med 1983; 75: 241-8.

6. Sato T, Nishinaga $M$, Kawamoto A, Ozawa $T$, Takatsuji $H$. Accuracy of the continuous blood pressure monitor based on arterial tonometry. Hypertension 1993; 21: 866-74.

7. Sellers RD, Levy MJ, Amplatz $\mathrm{K}$, Lillehei CW. Left retrograde cardioangiography in acquired cardiac disease. Am J Cardiol 1964; 14: 437-47.

8. Cohen MV, Gorlin R. Modified orifice equation for the calculation of mitral valve area. Am Heart J $1972 ; 84: 839-40$.

9. Hatle L, Angelsen B, Tromsdal A. Noninvasive assessment of atrioventricular pressure half-time by Doppler ultrasound. Circulation 1979; 60: 1096-104.

10. Nanda NC. Mitral valve. In: Nanda NC, editors. Atlas of Color Doppler Echocardiography. Philadelphia: Lea \& Febiger, 1989: 46-55.

11. Knowles JF, Gorlin R, Storcy GF. Clinical test for pulmonary congestion with use of the Valsalva maneuver. JAMA 1956; 160: 44-8.

12. Hamilton WF, Woodbury RA, Harper HT. Physiologic relations between intrathoracic, intraspinal, and arterial pressures. JAMA 1936; 107: 853-57.

13. Shimada K, Kitazumi $T$, Ogura $H$, Sadakane N, Ozawa $T$. Effects of age and blood pressure on the cardiovascular responses to the Valsalva maneuver. J Am Geriatr Soc 1986; 34: 431-4.

14. Zema MJ, Restivo B, Sos T, Sniderman KW, Kline S. Left ventricular dysfunction-bed side Valsalva maneuver. Br Heart J 1980; 44: 560-9.

15. Gorlin R, Knowles JH, Clifford C, Storey F. The Valsalva maneuver as a test of cardiac function. Am J Med 1957; 22; 197-212.

16. Shimada $\mathrm{K}$, Kitazumi $\mathrm{T}$, Sadakane N, Ogura $\mathrm{H}$, Ozawa T. Age-related changes of baroreflex function, plasma norepinephrine, and blood pressure. Hypertension 1985; 7: 113-7.

17. Schmidt D, Shah PK. Accurate detection of elevared left ventricular filling pressure by a simplified bedside application of the Valsalva maneuver. Am J Cardiol 1993; 71: 462-5.

18. McIntyre KM, Vita JA, Lambrew CT, Freeman J, Loscalzo J. A noninvasive method of predicting pulmonary-capillary wedge pressure. New Engl J Med 1992; 327: 1715-20. 